\title{
Investigation on the low-temperature transformations of poly(furfuryl alcohol) deposited on MCM-41
}

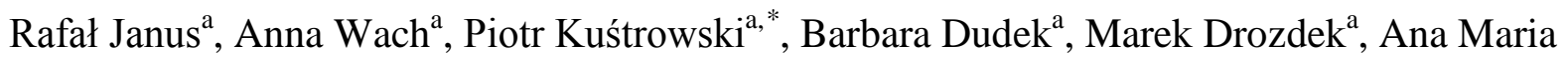

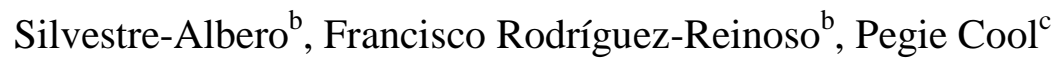

${ }^{a}$ Department of Chemical Technology, Faculty of Chemistry, Jagiellonian University, Ingardena 3, 30-060 Kraków, Poland

${ }^{b}$ Departamento de Química Inorgánica, Universidad de Alicante, Apartado 99, E-03080 Alicante, Spain

${ }^{c}$ Department of Chemistry, University of Antwerpen, Universiteitsplein 1, B-2610 Wilrijk, Belgium

\begin{abstract}
MCM-41-type mesoporous silica was used as a support for poly(furfuryl alcohol) deposition. This material was produced by precipitation-polycondensation of furfuryl alcohol (FA) in aqueous slurry of the $\mathrm{SiO}_{2}$ support followed by controlled partial carbonization. By tuning the FA/MCM-41 mass ratio in the reaction mixture, various amounts of polymer particles were introduced on the inner and outer surface of the MCM support. The thermal decomposition of the PFA/MCM-41 composites was studied by thermogravimetry (TG) and spectroscopic techniques (DRIFT, XPS), whereas the evolution of textural parameters with increasing polymer content was investigated using low-temperature adsorption of nitrogen. The mechanism of thermal transformations of PFA deposited on the MCM-41 surface was discussed in detail. It was found that heating at a temperature of about $523 \mathrm{~K}$ resulted in opening of the furan rings and the formation of $\gamma$-diketone moieties, which were found to be
\end{abstract}

\footnotetext{
${ }^{*}$ Corresponding author. Tel. +48-12-6632006; fax +48-12-6340515. E-mail address: kustrows@chemia.uj.edu.pl
} 
the highest effective surface species for the adsorption of polar volatile organic compounds. A further increase in calcination temperature caused a drop in the amounts of surface carbonyls and the appearance of condensed aromatic domains.

Keywords: MCM-41; Precipitation-polycondensation; Poly(furfuryl alcohol); Adsorption;

Volatile Organic Compounds

\section{Introduction}

Among many types of known adsorptive materials (zeolites, silica gels, clays, molecular sieves) [1-5], activated carbons (ACs) are widely used in commercial scale due to low costs of large-capacity production and desirable microstructural properties. The process of ACs manufacture consists of two essential steps: (i) carbonization of raw carbon-containing material under controlled (usually oxygen-free) conditions and (ii) physical or chemical activation resulting in a formation of eligible porosity and/or surface composition [6,7]. Activated carbons find a large number of applications, mainly in environmental protection (e.g. adsorption of volatile organic compounds (VOCs) [8-12] and $\mathrm{CO}_{2}$ [13-15] as well as purification of waste water by the removal of heavy metal ions [16] or phenols $[17,18]$ ), chemical industry (as a catalyst support) [19,20] and electrochemistry [21,22]. Many different raw materials are used for the fabrication of ACs, for instance: coal, charcoal, biomass or waste synthetic polymers [5,23-32]. The use of synthetic polymeric materials seems to be one of the most promising methods for the production of special quality activated carbons due to the possibility of tailoring and control of the properties of the final adsorbent as well as its high reproducibility. Obviously, the raw polymer used strictly determines the structural, textural and surface properties of the final carbon adsorbent. In this role, for example, 
phenolic resins, polyvinyl chloride, various acrylic polymers, and copolymers of divinylbenzene [33-35] have been tested.

Furfural, an aldehyde obtained as the hydrolysis product of pentosan-rich biomass, e.g. baggase, corn, birch wood or wheat [36], is one of the very promising, environmental-friendly and cheap initial base substances for the production of carbon adsorbents. This aldehyde can be easily transformed into furfuryl alcohol (FA), this being a monomer for the synthesis of poly(furfuryl alcohol) (PFA). PFA was found to be a useful polymer for the fabrication of high quality, reproducible and stable carbon-based, microporous adsorbents by pyrolysis and optionally subsequent modification [37-43]. Polyacrylonitrile (PAN), a high-melting and spinnable duroplast, is another extensively studied raw material for the production of carbon fibers, filaments and films [6,8,44-48].

In recent years a lot of attention has been focused on various hybrid carbon-inorganic materials that combine characteristics of both components $[6,8,40,41,43]$. Partially carbonized and properly functionalized organic compounds dispersed on porous silica supports were studied, for example, as catalysts for various industrial processes. Toda et al. [49] reported the application of incompletely carbonized sugars (D-glucose and sucrose) sulfonated with sulfuric acid as catalysts in the biodiesel production. Similar materials dispersed on SBA-15 were used in esterification of long-chain fatty acids and transesterification of soybean oil with methanol [50], liquid-phase Beckmann rearrangement of cyclohexanone oxime to $\varepsilon$ caprolactam, condensation of various aldehydes with ethylene glycol [51], as well as selective dimerization of $\alpha$-methylstyrene [52]. Furthermore, incompletely carbonized and sulfonated sucrose deposited on MCM-48 was successfully tested as a catalyst for esterification of higher fatty acids with ethanol [53].

Among various methods of modification of $\mathrm{SiO}_{2}$ surface, the introduction of organic species by atom transfer radical polymerization (ATRP) seems to be very interesting [54]. Poly-p- 
styrenesulfonic acid grafted by this route on SBA-15 silica appeared to be an active catalyst of esterification processes $[55,56]$. Although there are a few papers dealing with using the ATRP method for the modification of SBA-15, silica materials exhibiting narrower pores (e.g. MCM-41) have been much less studied.

Our goal is the investigation of the carbon surface, efficient in adsorption of VOCs, obtained by the deposition of polymeric species (PFA-derived) on mesoporous MCM-41 support, followed by partial carbonization, to avoid the diffusion limitations typical for commercial microporous ACs. MCM-41 with deposited carbon has smaller pores but larger than microporous PFA-based carbon alone and shows higher adsorption of polar volatile organic compound. On the other hand, the proposed synthesis route allows to omit the complicated methods of mesoporous PFA-based carbon synthesis reported earlier $[57,58]$. In this work, we propose a new, simplified method for the synthesis of poly(furfuryl alcohol)-derived carbon supported on mesoporous MCM-41 silica molecular sieve. Various amounts of polymer were introduced by acid-catalyzed precipitation-polycondensation of FA in aqueous slurry of the support, followed by partial carbonization. Thermally degraded PFA supported on the mesoporous silica appeared to be an efficient adsorbent of methyl-ethyl ketone (MEK) vapour. To understand the role of the different types of surface species, formed during PFA decomposition, on the adsorption capacity of MEK we investigated the mechanism of lowtemperature transformations of bulky PFA and silica-supported polymer. For this purpose, thermogravimetry coupled with IR (TG/DTG/FT-IR), DRIFT and XPS measurements were performed. 


\section{Experimental}

\subsection{Synthesis}

Mesoporous MCM-41 molecular sieve was synthesized using a molar gel composition: 1.00 tetraethylorthosilicate (TEOS): 0.16 hexadecyltrimethylammonium chloride $\left(\mathrm{C}_{16} \mathrm{TMACl}\right)$ : 2.76 $\mathrm{NH}_{3}$ : $140.13 \mathrm{H}_{2} \mathrm{O}$. Amounts of $525 \mathrm{ml}$ of distilled water, $45.3 \mathrm{ml}$ of $\mathrm{C}_{16} \mathrm{TMACl}$ (25\% water solution, Aldrich) and $44 \mathrm{ml}$ of ammonia solution (25\%, Polish Chemical Reagents) were mixed in a $1000 \mathrm{ml}$ beaker and stirred for $30 \mathrm{~min}$ at room temperature. Then, $48.6 \mathrm{ml}$ of TEOS (98\%, Aldrich) was added dropwise. Next, the resulting white suspension was stirred for $1 \mathrm{~h}$. Subsequently, the product was filtered, washed with distilled water and dried at $295 \mathrm{~K}$ for $48 \mathrm{~h}$. Finally, the organic template was removed by calcination of the material at $825 \mathrm{~K}$ for $8 \mathrm{~h}$ at a heating rate of $1 \mathrm{~K} / \mathrm{min}$. The low-angle XRD pattern of calcined MCM-41 shows the characteristics of a highly ordered hexagonal structure (p6mm) with $a$ cell parameter equal to $3.81 \mathrm{~nm}$. The isotherm of $\mathrm{N}_{2}$ adsorption confirms obtaining well-defined MCM-41 structure with the BET surface area equal to $1037 \mathrm{~m}^{2} / \mathrm{g}$ and the total pore volume of $0.87 \mathrm{~cm}^{3} / \mathrm{g}$, whereas the mean pore size is about $3.7 \mathrm{~nm}$ (Supplementary information, Fig. S1 and S2).

PFAx/MCM-41 (where $x=0.1,0.4,1.0$ and 2.0 means an intended PFA/SiO ${ }_{2}$ mass ratio) composite materials were synthesized by acid-catalyzed precipitation polycondensation of furfuryl alcohol (FA) in aqueous slurry of MCM-41 support. Typically, $1.0 \mathrm{~g}$ of freshly calcined support was introduced into a three-necked flask (250 ml) equipped with a reflux condenser and placed in an oil bath on a magnetic stirrer. Then, adequate volumes of water and FA (98\%, Acros Organics) were added to obtain a total mixture volume of $140 \mathrm{ml}$. The intended $\mathrm{PFA} / \mathrm{SiO}_{2}$ mass ratios were achieved by adding appropriate amount of FA. The mixture was stirred for $0.5 \mathrm{~h}$ at room temperature. Subsequently, the proper volume of $\mathrm{HCl}$ (33\%, Polish Chemical Reagents), being an acid catalyst of polycondensation, was added. The $\mathrm{HCl} / \mathrm{FA}$ molar ratio was kept at a constant level of 6 . The polycondensation process was 
performed at $373 \mathrm{~K}$ for $6 \mathrm{~h}$. The obtained dark-brown composite was isolated by filtration, washed with distilled water and dried at $333 \mathrm{~K}$ overnight. Eventually, the PFA/MCM-41 precursors were partially carbonized in a tubular furnace at the temperature range of 423$1023 \mathrm{~K}\left(\mathrm{~N}_{2} ; 40 \mathrm{ml} / \mathrm{min}\right.$; a heating rate of $5 \mathrm{~K} / \mathrm{min}$ and an isothermal period of $\left.4 \mathrm{~h}\right)$. The thermally treated samples are labelled as calcined-PFAx/MCM-41.

Reference polymer materials were synthesized by the same procedure without using a silica support.

\subsection{Characterization}

Powder X-ray diffraction measurements were performed using Bruker D2 Phaser equipped with a LYNXEYE detector in the $2 \theta$ angle range of 0.8-5.0 . A JEM (JEOL) 2010 transmission electron microscope equipped with an INCA Energy TEM 100 analyzer and SIS MegaView II camera was used to collect TEM images. The used accelerating voltage was 200 $\mathrm{kV}$; a sample was placed on a copper holder coated with LASEY carbon film.

The textural parameters of molecular sieve MCM-41 and thermally transformed samples were determined based on the low temperature $(77 \mathrm{~K})$ nitrogen adsorption-desorption isotherms. The isotherms were collected in a home-made fully automated equipment designed and constructed by the Advanced Materials group (LMA), commercialized as N2Gsorb-6 (Gas to Materials Technologies). The samples were preliminary outgassed at 473 for $4 \mathrm{~h}$ at a base pressure of $1.3 \cdot 10^{-3} \mathrm{~Pa}$. The dried PFA/MCM-41 composites were analyzed by thermogravimetric method (TG) in an oxidizing or inert atmosphere (air $(100 \mathrm{ml} / \mathrm{min}$ ) or nitrogen (20 ml/min), ca. $5 \mathrm{mg}$ of sample placed in a corundum crucible, outgassing at room temperature in flowing gas for $1 \mathrm{~h}$, temperature range $=303-1273 \mathrm{~K}$, heating rate $=20 \mathrm{~K} / \mathrm{min}$ ) using a SDT Q600 apparatus (TA Instruments). The composition of the volatile products evolved during thermal decomposition of PFA/MCM-41 precursor in an inert atmosphere was 
investigated using TG/FT-IR interface (Thermo Scientific) connected on-line with Nicolet 6700 FTIR spectrometer. The spectra were collected in a region of $400-4000 \mathrm{~cm}^{-1}$ at a resolution of $4 \mathrm{~cm}^{-1}$ and a time interval of $3 \mathrm{~s}$.

The PFA/MCM-41 precursors and the samples decomposed in the temperature range of 473$673 \mathrm{~K}$ were examined by DRIFT and XPS. Prior to the DRIFT measurements the samples were diluted to $2 \%$ by weight in potassium bromide and softly milled in an agate mortar. The mid infrared spectra (200 scans each) were collected on a Nicolet 6700 FTIR (Thermo Scientific) spectrometer at a resolution of $4 \mathrm{~cm}^{-1}$. The XPS measurements were performed with a Prevac photoelectron spectrometer equipped with a hemispherical analyzer VG SCIENTA R3000. The spectra were taken using a monochromatized aluminum source AlK $\alpha$ ( $E=1486.6 \mathrm{eV}$ ) and a low energy electron flood gun (FS40A-PS) to compensate charge accumulation on the surface of nonconductive samples. The base pressure in the analytical chamber was $5 \cdot 10^{-9}$ mbar. The surface composition was studied based on the areas and binding energies of $\mathrm{C} 1$ s, O $1 s$ and Si $2 p$ core levels. The binding energy values of measured regions were referenced to the Si $2 p$ core level $(103.6 \mathrm{eV})$.

\subsection{Adsorption tests}

An amount of ca. $50 \mathrm{mg}$ of sample was weighted in a stainless steel sample cell with a total volume of $1.2 \mathrm{ml}$. The sample cell was placed in an oven. The constant flow of carrier gas $\left(\mathrm{N}_{2}, 20 \mathrm{ml} / \mathrm{min}\right.$ ) was passed through the adsorbent bed and the cell was heated up to $423 \mathrm{~K}$ for $0.5 \mathrm{~h}$ (an outgassing step). Then, the sample cell was weighted again in order to determine the exact mass of outgassed sample and placed back in the oven kept at $313 \mathrm{~K}$. Subsequently, the flow of carrier gas $\left(\mathrm{N}_{2}, 20 \mathrm{ml} / \mathrm{min}\right)$, saturated with methyl-ethyl ketone vapor, was passed by the adsorbent at $323 \mathrm{~K}$ until the flame ionization detector (FID) signal reached a plateau. In the next step, the dosage of MEK was interrupted and the system was purged with pure carrier 
gas in order to remove the loosely adsorbed forms of ketone. Then, the sample cell was heated from 323 up to $523 \mathrm{~K}$ at a heating rate of $15 \mathrm{~K} / \mathrm{min}$ in a flow of nitrogen $(20 \mathrm{ml} / \mathrm{min})$. No clear decomposition of the composite calcined at $473 \mathrm{~K}$ was detected during the TPD measurement in the temperature range of 473-523 K. The adsorption capacities of the studied samples were calculated from the FID signal based on the calibration of the detector response by injections of various amounts of liquid MEK.

\section{Results and discussion}

\subsection{Effectiveness of deposition of PFA on MCM-41 surface}

The real content of poly(furfuryl alcohol) introduced onto the MCM-41 support was determined by thermogravimetric measurements performed in the oxidizing atmosphere. The TG/DTG curves as well as the polymer/silica mass ratios, calculated from the mass losses detected above $393 \mathrm{~K}$, are shown in Fig. 1. It is found that the real PFA loadings are lower than the intended values for all the studied samples. Obviously, the higher concentration of FA in the reaction slurry provided more effective deposition onto the silica support. The efficiency of PFA deposition is equal to 39.0, 54.3, 34.0 and $60.7 \%$ for the expected PFA/support ratios of $0.1,0.4,1.0$ and 2.0, respectively. This means that the real PFA/MCM41 mass ratios of $0.04,0.22,0.34$ and 1.21 , respectively, were achieved. During polycondensation the PFA species are formed on the inner and outer surface of the MCM-41 support as well as outside the silica particles. When the oligomeric molecule reaches the critical molecular mass, it ceases to be water soluble and it precipitates on the silica surface. A part of monomer molecules is grafted on the surface silanol groups and therefore 'surfaceanchored' polymer is also formed. However, the isolation of solid materials from the reaction medium after the syntheses leads to leaching short-chain, water-soluble oligomers resulting in a decrease in the deposition yield. 
The distribution of deposited PFA on the MCM-41 support was investigated by collecting low-temperature $\mathrm{N}_{2}$ adsorption isotherms for the materials after partial carbonization at $523 \mathrm{~K}$. The measured isotherms and pore size distributions calculated with the NLDFT equilibrium model are comparatively shown in Fig. 2. The amount of adsorbed nitrogen drops with an increase in the content of PFA-derived species. The changes in the shape of the isotherms in a $\mathrm{p} / \mathrm{p}_{0}$ range of about $0.2-0.3$ suggest the reduction of the volume of accessible mesopores caused by their gradual blocking with the increase of the polymer content. For the calcined-PFA2.0/MCM-41 material, the recorded isotherm with a loss of the capillary condensation step is typical for a poorly porous solid, revealing that the formed polymer totally filled the mesopore system and/or blocked the pore mouths. Furthermore, the deposition of polymer species on the MCM-41 surface followed by partial carbonization resulted in a gradual decrease in the pore size (Fig. 2B) as well as the BET surface area and total pore volume (Fig. 3 and Table 1S). A linear correlation between the PFA content and the total pore volume of the material was found. Extrapolation of these values for $V_{\text {total }} \rightarrow 0$ allows us to conclude that the blockage of the pore system by formed polymer would be observed at the real PFA/MCM-41 mass ratio between 0.5 and 0.6. In spite of mouths of a few pores can be blocked by the PFA particles, the chemical composition and textural properties as well as the collected TEM images (Fig. 4) confirm homogeneous dispersion of precipitated PFA on the complete surface (both outer and inner) of the silica support. The phenomenon of gradual filling of the silica mesochannels with organic species was reported earlier by several authors $[59,60]$. The described approach to the calculation of the polymer layer thickness inspired us to determine the thickness of PFA film deposited on the MCM-41 surface. Fig. S3 (see Supplementary information) shows the results obtained from the difference in the pore size of MCM-41 before and after deposition of PFA (determined by NLDFT) correlated with the real polymer/MCM-41 mass ratio (determined by TG). 


\subsection{Adsorption capacity of partially carbonized PFA/MCM-41 composites}

The synthesized calcined-PFA/MCM-41 composites were tested as adsorbents of methylethyl ketone vapor chosen as a representative molecule of polar derivatives of hydrocarbons. The measurements were performed in the dynamic adsorption system with the determination of amount of MEK adsorbed by temperature-programmed desorption (TPD). It was found that two parameters, namely the content of carbonaceous species and temperature of thermal treatment, affect strongly the adsorption properties of the calcined-PFA/MCM-41 composites. We observed that the MCM-41 modified with small amounts of PFA exhibits higher adsorption capacity compared to the pristine silica (Fig. 5B). On the contrary, the introduction of larger amounts of polymer species into the pore system of MCM-41 support results in blocking of the pore system and ipso facto reduction of the accessibility of the inner adsorption centers, as is confirmed by the textural parameters (Fig. 3 and Table 1). Therefore, we chose the PFA0.4/MCM-41 sample to investigate the influence of temperature of the heat treatment on the adsorption capacity (Fig. 5A). It can be seen that the calcination temperature plays a crucial role in tuning the adsorption properties. It is noticed that the material calcined at $523 \mathrm{~K}$ shows the best adsorption capacity of $0.29 \mathrm{~g}_{\mathrm{MEK}} / \mathrm{g}_{\mathrm{ads}}$, which is about $26 \%$ higher than that measured for the unmodified support. It should be noticed that the adsorption capacity of bulky PFA calcined at the same temperature is below the detection limit. The unsupported polymer exhibits an increase in the adsorption efficiency after carbonization at temperatures above $700 \mathrm{~K}$, while an opposite effect is observed for the composite samples. This fact suggests that bulky PFA calcined at elevated temperatures forms a type of carbon microstructure that is efficient in the removal of organic molecules from the gas phase. The low-temperature adsorption of $\mathrm{N}_{2}$ showed (cf. Fig. S4, Supplementary information) that the bulky PFA becomes a porous material after carbonization at temperature of $773 \mathrm{~K}$ or higher.

On the other hand, in the case of the PFA/MCM-41 composites the appearance of specific 
surface species exhibiting a high affinity to MEK is expected after the thermal treatment at $523 \mathrm{~K}$.

\subsection{Thermal stability of PFA deposited on MCM-41 support}

In order to recognize the mechanism of thermal decomposition of deposited polymer, bulky PFA and the PFA/MCM-41 composites were studied by thermal analysis in inert atmosphere. The collected thermograms are shown in Fig. 6A. Moreover, the corresponding FTIR maps of the evolved gases for bulky PFA, PFA0.4/MCM-41 and PFA1.0/MCM-41 are illustrated in Fig. 6B.

Moisture is removed from the sample surface at low temperature (below $400 \mathrm{~K}$ ). The essential decomposition of the samples, unsupported PFA and PFA/MCM-41, starts in inert atmosphere at about $400 \mathrm{~K}$ and proceeds in two overlapping stages. The first mass loss of bulky PFA, with a DTG maximum at about $630 \mathrm{~K}$, is connected with the emission of $\mathrm{CO}_{2}$ (absorption bands at 2305 and $2360 \mathrm{~cm}^{-1}$ in the FTIR map), small amounts of CO (weak bands at 2170 and $2110 \mathrm{~cm}^{-1}$ ) and $\mathrm{H}_{2} \mathrm{O}$ (broad bands at $1300-1800$ and $3500-4000 \mathrm{~cm}^{-1}$ ). The absorption bands characteristic for carbonyl species $\left(1700 \mathrm{~cm}^{-1}\right)$ and ether linkage $\left(1020 \mathrm{~cm}^{-1}\right)$ are also observed in the FTIR spectra of gases evolved in this temperature range. Guigo et al. [36] ascribed such carbonyl groups to the formation of acetone, butan-2-one and pentan-2-one due to the scissions of furan ring and methylene. The second decomposition step, with a maximum at $710 \mathrm{~K}$, corresponds to the emission of the $\mathrm{CO}_{\mathrm{x}}, \mathrm{H}_{2} \mathrm{O}$ and $\mathrm{CH}_{4}$ (weak absorption bands at about 3017 and $1300 \mathrm{~cm}^{-1}$ ) [61]. At temperatures above $710 \mathrm{~K}$ the bands ascribed to $\mathrm{H}_{2} \mathrm{O}, \mathrm{CO}_{\mathrm{x}}$ and $\mathrm{CH}_{4}$ are still detected, but the rate of mass loss gradually decreases.

It is interesting to see that the decomposition of supported poly(furfuryl alcohol) shows a higher mass loss compared to bulky PFA when normalized to the real content of polymer in the studied material. For example, in the case of PFA1.0/MCM-41 96.2\% of polymer mass 
loss is observed over the entire temperature range, whereas for pure PFA only $54.5 \%$ of its initial mass is lost. Moreover, there are differences in the mechanism of polymer decomposition. For PFA0.4/MCM-41 and PFA1.0/MCM-41, all the decomposition stages are widely overlapping. Obviously, the FTIR band intensities for PFA0.4/MCM-41 are weaker compared to the PFA1.0/MCM-41 sample in the whole temperature range due to the lower content of organic species (cf. Fig. 6B). In the first decomposition step, the emission of $\mathrm{CO}_{\mathrm{x}}$ and $\mathrm{H}_{2} \mathrm{O}$ starts at lower temperature both the PFA/MCM-41 composites than for unsupported PFA (cf. Fig. 6B). In the subsequent step, starting above $540 \mathrm{~K}$, the emission of small amounts of methane is found. This effect is much weaker compared to that observed for the bulk polymer. Contrary to the bulky PFA, the emission of $\mathrm{CO}_{\mathrm{x}}$ and $\mathrm{H}_{2} \mathrm{O}$ at higher temperatures (above $700 \mathrm{~K}$ ) is kept on a constant level. It can be therefore concluded that PFA supported on MCM-41 exhibits lower thermal stability regardless of the polymer content in the composite. This effect, probably caused by the relatively easy furan ring-opening occurring during the decomposition of polymer deposited on the silica surface, is opposite to that reported by Guigo et al. [36]. However, the thermal stability of the hybrid PFA/silica materials discussed in [36] cannot be simply compared to that of the composites presented herein. The samples studied by Guigo et al. [36] were prepared by simultaneous inorganic mesophase formation and furfuryl alcohol polycondensation. Finally, they obtained materials composed of nanometric clusters branched together through condensation reaction to form the 3D organic-inorganic network. In our synthesis, PFA is introduced by the post-synthesis modification of MCM-41: the prepared silica support is gradually coated with the 2D PFA layers by the precipitation polycondensation of furfuryl alcohol in a water slurry of silica. Thus, Guigo et al. [36] discussed the thermal behavior of non-porous, bulky sample, whereas our composites exhibit porosity. It should be therefore assumed that the bulky PFA/silica 
composite may show some differences in the thermal stability compared to the thin layers of PFA deposited on the mesoporous silica support as we proved.

\subsection{Mechanism of PFA decomposition}

A type of polymeric species evolved on the mesoporous silica was identified by spectroscopic techniques. The DRIFT spectra collected for bulky PFA as well as the PFA0.4/MCM-41 and PFA1.0/MCM-41 composites calcined at elevated temperatures showed that many of the absorption bands characteristics for PFA are still present (Fig. S5, Supplementary information).

The main characteristic peaks for neat PFA (Fig. S5, A) are observed at $3400 \mathrm{~cm}^{-1}(-\mathrm{OH}$ stretching), $3119 \mathrm{~cm}^{-1}$ (-CH in aromatic rings), 2970, 2921 and $2850 \mathrm{~cm}^{-1}$ (aliphatic $-\mathrm{CH}$ and $-\mathrm{CH}_{2}$ stretching vibrations), $1600 \mathrm{~cm}^{-1}$ (aromatic $\mathrm{C}=\mathrm{C}$ ), 1550 and $1500 \mathrm{~cm}^{-1}$ (furan ring vibrations), $1425 \mathrm{~cm}^{-1}$ (asymmetric bending $\mathrm{CH}_{2}$ vibrations), $1355 \mathrm{~cm}^{-1}$ (-CH furan ring deformation), $1100 \mathrm{~cm}^{-1}$ (C-O stretching), $1020 \mathrm{~cm}^{-1}$ (asymmetric and symmetric $=\mathrm{C}-\mathrm{O}-\mathrm{C}=$ vibrations in 2-substituted furan ring) and $795 \mathrm{~cm}^{-1}$ (the vibrations of $\mathrm{C}-\mathrm{C}$ bonds in 2,5disubstituted furan rings forming the polymer chain). A sharp, intensive band at $1709 \mathrm{~cm}^{-1}$ indicates the presence of carbonyl groups (stretching vibrations). These groups appear as a result of acid-catalyzed ring opening of furan rings [62]. In this process, $\gamma$-diketone species are formed by the electrophilic attack of water molecules being a by-product of FA polycondensation on furan ring in the polymer chain. Increasing the heat treatment temperature to 523-673 K we find the main differences in the region of carbonyl $\left(1709 \mathrm{~cm}^{-1}\right)$ and aliphatic $-\mathrm{CH}$ and $-\mathrm{CH}_{2}$ stretching vibrations (2970, 2921 and $2850 \mathrm{~cm}^{-1}$ ). The relative intensity of these bands gradually decreases, while the intensity of aromatic $C=C$ band (1600 $\mathrm{cm}^{-1}$ ) clearly increases. These effects are due to the decomposition of oxygen-containing groups connected with loss of carbon oxides and water resulting in the formation of 
condensed aromatic species. Thus, we can conclude that polyaromatic domains and partially decomposed polymer chains coexist in the samples thermally treated in the temperature range of 523-673 K.

The DRIFT spectrum collected for both PFA0.4/MCM-41 and PFA1.0/MCM-41 composites demonstrates the presence of characteristic bands of both the components, silica and poly(furfuryl alcohol) (Fig. S5, B and C). The sharp band at $3744 \mathrm{~cm}^{-1}$ is ascribed to the stretching vibrations of terminal surface silanol groups, the broad band at $3000-3750 \mathrm{~cm}^{-1}$ proves the presence of hydrogen bridges between the adjacent silanol groups. It is noteworthy that in case of the sample decorated with low PFA amount the intensity of the band at 3744 $\mathrm{cm}^{-1}$ increases with the calcination temperature, while the decrease in the intensity of the band at $3000-3750 \mathrm{~cm}^{-1}$ is observed. This effect is ascribed to the decay of hydrogen bridges between adjacent silanol surface groups and a consequent formation of terminal silanol groups. The bands at $1000-1250 \mathrm{~cm}^{-1}$ and $800 \mathrm{~cm}^{-1}$ are assigned to $\mathrm{Si}-\mathrm{O}$ stretching vibrations, whereas the absorption at $963 \mathrm{~cm}^{-1}$ corresponds to $\mathrm{Si}-\mathrm{OH}$ stretching vibrations. The unexpected evolution of the aliphatic stretching $-\mathrm{CH}$ and $-\mathrm{CH}_{2}\left(2970,2921\right.$ and $\left.2850 \mathrm{~cm}^{-1}\right)$ is noticed. As seen, the intensity of these bands increases significantly for the composite calcined at temperature up to $523 \mathrm{~K}$. We ascribed this effect to opening the furan ring and the formation of $\gamma$-diketone moieties. The thermal treatment at higher temperature causes a gradual decrease in amounts of aliphatic carbon and deeper carbonization connected with the creation of higher condensed polyunsaturated graphite-like species [63-68]. This conclusion is strongly supported by the foregoing TG-FTIR studies.

The TG and FITR results are also reflected in the XPS spectra. The XPS spectrum collected for the PFA0.4/MCM-41 composite after heat treatment at $473 \mathrm{~K}$ (Fig. S6, Supplementary information) shows changes in the distribution of species present on the composite's surface (Table 1). A slight decrease in the amount of silicon dioxide is measured, which is caused by 
the appearance of more condensed, dehydrated and partially degraded PFA covering the inorganic support and hindering the $\mathrm{X}$-ray penetration. The highest content of $\mathrm{C}=\mathrm{O}$ species is detected after the thermal treatment at $523 \mathrm{~K}$. A raise of the calcination temperature to 573$673 \mathrm{~K}$ leads to further structural transformations of the polymer. Thus, a decrease in oxygen amount, related to presence of carbonyl groups and furan rings, is observed, additionally, furan rings begin to coalescence generating a condensed structure.

Taking into account the results of TG/DTG/FTIR, DRIFT and XPS measurements we propose the mechanism of the formation of carbonyl moieties during the synthesis at $373 \mathrm{~K}$ and further thermal transformation of PFA deposited on the MCM-41 silica as shown in Fig. 7. Evidently, the carbonyl groups existing on the sample surface can be considered as the most effective in the adsorption of MEK molecules. The maximum concentration of these carbonyl groups determined by the aforementioned methods was observed after the calcination at $523 \mathrm{~K}$. Therefore, the increase in the thermal treatment temperature above $523 \mathrm{~K}$, causing the effect of surface $\mathrm{C}=\mathrm{O}$ decomposition, is undesirable. An active role of carbonyl species in the MEK adsorption was confirmed earlier by Monte Carlo simulations [69]. This effect was explained by the charge-dipole interactions, which are attributed to the high dipole moment of the MEK molecule, as well as the charge-induced dipole interactions due to the high polarizability of the MEK molecule.

\section{Conclusion}

The precipitation-polymerization is found to be an effective method of homogeneous introduction of poly(furfuryl alcohol) on the inner and outer surface of mesoporous MCM-41. The amount of deposited polymer can be controlled by furfuryl alcohol/ $\mathrm{SiO}_{2}$ mass ratio in the reaction mixture. Supported PFA exhibits significantly lower thermal stability compared to bulky polymer. It undergoes complex decomposition leading to condensed aromatic domains 
via opening of furan rings and the formation of $\gamma$-diketone moieties. These carbonyl species, with the highest surface concentration after calcination at $523 \mathrm{~K}$, are responsible for the high sorption capacity of calcined-PFA/MCM-41 adsorbents in the removal of polar volatile organic compounds, such as methyl-ethyl ketone. MCM-41 decorated with partially carbonized PFA layers exhibits better sorption capacity of MEK compared to microporous PFA-based carbon.

\section{Acknowledgement}

This work was supported by the Polish Ministry of Science and Higher Education under the grant no. N N507 553238. Rafał Janus wishes to thank the Foundation for Polish Science MPD Programme co-financed by the EU European Regional Development Fund for the financial support. The research was carried out with equipment purchased thanks to financial help from the European Regional Development Fund within the framework of the Polish Innovation Economy Operational Program (contract no. POIG.02.01.00-12-023/08).

\section{Supplementary Information Available}

Nine figures: one with the XRD pattern of pristine MCM-41, one with the experimental nitrogen adsorption data for MCM-41, one with the PFA layer thickness as a function of real polymer/MCM-41 mass ratio, one with the $\mathrm{N}_{2}$ adsorption isotherms collected for bulky PFA carbonized at different temperatures, five with the DRIFT and XPS spectra for the studied composites. One table with textural parameters of the studied samples. 


\section{References}

(1) Pires, J.; Carvalho, A.; Veloso, P.; Carvalho, M. B. Preparation of dealuminated faujasites for adsorption of volatile organic compounds. J. Mater. Chem. 2002, 12, 31003104.

(2) Zaitan, H.; Bianchi, D.; Achak, O.; Chafik, T. A comparative study of the adsorption and desorption of o-xylene onto bentonite clay and alumina. J. Hazard. Mater. 2008, 153, 852-859.

(3) Hung, C. T.; Bai, H. Adsorption behaviors of organic vapors using mesoporous silica particles made by evaporation induced self assembly method. Chem. Eng. Sci. 2008, 63, 1997-2005.

(4) Hung, C.T.; Bai, H.; Karthik, M. Ordered mesoporous silica particles and Si-MCM-41 for the adsorption of acetone: a comparative study. Sep. Purif. Technol. 2009, 64, 265-272.

(5) Gregg, S. J.; Sing, K. S. W. Adsorption, Surface Area and Porosity. Academic Press, London, New York, 1982.

(6) Kuśtrowski, P.; Janus, R.; Kochanowski, A.; Chmielarz, L.; Dudek, B.; Piwowarska, Z.; Michalik, M. Adsorption properties of carbonized polyacrylonitrile deposited on $\gamma$-alumina and silica gel by precipitation polymerization. Mater. Res. Bull. 2010, 45, 787-793.

(7) Marsh, H.; Rodríguez-Reinoso, F. Activated Carbon. Elsevier Science \& Technology Books, London, 2006.

(8) Janus, R.; Kuśtrowski, P.; Dudek, B.; Piwowarska, Z.; Kochanowski, A.; Michalik, M.; Cool, P. Removal of methyl-ethyl ketone vapour on polyacrylonitrile-derived carbon/mesoporous silica nanocomposite adsorbents. Micropor. Mesopor. Mater. 2011, 145, 65-73. 
(9) Saini, K. V.; Andrade, M.; Pinto, M. L.; Carvalho, A. P.; Pires, J. How the adsorption properties get changed when going from SBA-15 to its CMK-3 carbon replica. Sep. Purif. Technol. 2010, 75, 366-376.

(10) Dąbrowski, A.; Podkościelny, P.; Hubicki, Z.; Barczak. M. Adsorption of phenolic compounds by activated carbons - critical review. Chemosphere 2005, 58, 1049-1070.

(11) Silvestre-Albero, A.; Ramos-Fernández, J. M.; Martínez-Escandell, M.; SepúlvedaEscribano, A.; Silvestre-Albero, J.; Rodríguez-Reinoso. F. High saturation capacity of activated carbons prepared from mesophase pitch in the removal of volatile organic compounds. Carbon 2010, 48, 548-556.

(12) Lillo-Ródenas, M. A.; Cazorla-Amorós, D.; Linares-Solano, A. Benzene and toluene adsorption at low concentration on activated carbon fibres. Adsorption 2011, 17, 473-481.

(13) Sevilla, M.; Fuertes, A. B. $\mathrm{CO}_{2}$ adsorption by activated templated carbons. J. Colloid Interface Sci. 2012, 366, 147-154.

(14) Chandrasekar, G.; Son, W. J.; Ahn, W. S. Synthesis of mesoporous materials SBA-15 and CMK-3 from fly ash and their application for $\mathrm{CO}_{2}$ adsorption. J. Porous. Mater. 2009, 16, 545-551.

(15) Silvestre-Albero, J.; Wahby, A.; Sepulveda-Escribano, A.; Martínez-Escandell, M.; Kaneko, K.; Rodríguez-Reinoso, F. Ultrahigh $\mathrm{CO}_{2}$ adsorption capacity on carbon molecular sieves at room temperature. Chem. Commun. 2011, 47, 6840-6842.

(16) Ahn, Ch. K.; Park, D.; Woo, S. H.; Park, J. M. Removal of cationic heavy metal from aqueous solution by activated carbon impregnated with anionic surfactants. J. Hazard. Mater. 2009, 164, 1130-1136. 
(17) Velasco, L. F.; Tsyntsarski, B.; Petrova, B.; Budinova, T.; Petrov, N.; Parra, J. B.; Ania, C. O. Carbon foams as catalyst supports for phenol photodegradation. J. Hazard. Mater. 2010, 184, 843-848.

(18) He, J.; Ma, K.; Jin, J.; Dong, Z.; Wang, J.; Li, R. Preparation and characterization of octyl-modified ordered mesoporous carbon CMK-3 for phenol adsorption. Micropor. Mesopor. Mater. 2009, 121, 173-177.

(19) Calvillo, L.; Cellorio, V.; Moliner, R.; Lázaro, M. J. Influence of the support on the physicochemical properties of Pt electrocatalysts: Comparison of catalysts supported on different carbon materials. Mater. Chem. Phys. 2011, 127, 335-341.

(20) Salgado, J. R. C.; Alcaide, F.; Álvarez, G.; Calvillo, L.; Lázaro, M. J. Pt-Ru electrocatalysts supported on ordered mesoporous carbon for direct methanol fuel cell. $J$. Power Sources 2010, 195, 4022-4029.

(21) Zhang, H.; Tao, H.; Jiang, Y.; Jiao, Z.; Wu, M.; Zhao, B. Ordered CoO/CMK-3 nanocomposites as the anode materials for lithium-ion batteries. J. Power Sources 2010, 195, 2950-2955.

(22) Yang, M.; Gao, Q. Copper oxide and ordered mesoporous carbon composite with high performance using as anode material for lithium-ion battery. Micropor. Mesopor. Mater. 2011, 143, 230-235.

(23) Yue, Z.; Economy, J.; Bordson, G. Preparation and characterization of $\mathrm{NaOH}$-activated carbons from phenolic resin. J. Mater. Chem. 2006, 16, 1456-1461.

(24) Nakagawa, K.; Mukai, S. R.; Tamura, K.; Tamon, H. Mesoporous activated carbons from phenolic resins. Chem. Eng. Res. Des. 2007, 85, 1331-1337. 
(25) Tennison, S. R. Phenolic-resin-derived activated carbons. Appl. Catal., A 1998, 173, 289-311.

(26) Miyake, T.; Hanaya, M. Carbon-coated material with bimodal pore-size distribution. $J$. Mater. Sci. 2002, 37, 907-910.

(27) Chiang, H. L.; Chiang, P. C.; Chiang, Y. C.; Chang, E. E. Diffusivity of microporous carbon for benzene and methyl-ethyl ketone adsorption. Chemosphere 1999, 38, 2733-2746.

(28) Przepiórski, J.; Tryba, B.; Morawski, A. W. Adsorption of carbon dioxide on phenolic resin-based carbon spheres. Appl. Surf. Sci. 2002, 196, 296-300.

(29) Kim, M. I.; Yun, C. H.; Kim, Y. J.; Park, C. R.; Inagaki, M. Changes in pore properties of phenol formaldehyde-based carbon with carbonization and oxidation conditions. Carbon 2002, 40, 2003-2012.

(30) Leboda, R.; Skubiszewska-Zięba, J.; Tomaszewski, W.; Gun'ko, V. M. Structural and adsorptive properties of activated carbons prepared by carbonization and activation of resins. J. Coll. Interface Sci. 2003, 263, 533-541.

(31) Chen, X.; Jeyaseelan, S.; Graham, N. Physical and chemical properties study of the activated carbon made from sewage sludge. Waste Manage. 2002, 22, 755-760.

(32) Yardim, M. F.; Budinova, T.; Ekinci, E.; Petrov, N.; Razvigoroba, M.; Minkova, V. Removal of mercury(II) from aqueous solution by activated carbon obtained from furfural. Chemosphere 2003, 52, 835-841.

(33) Sedghi, A.; Farsani, R. E.; Shokuhfar, A. The effect of commercial polyacrylonitrile fibers characterizations on the produced carbon fibers properties. J. Mater. Process. Technol. 2008, 198, 60-67. 
(34) Gierszal, K. P.; Jaroniec, M.; Kim, T.-W.; Kim, J.; Ryoo, R. High temperature treatment of ordered mesoporous carbons prepared by using various carbon precursors and ordered mesoporous silica templates. New J. Chem. 2008, 32, 981-993.

(35) Hirano, Sh. I.; Ozawa, M.; Naka, Sh. Formation of non-graphitizable isothropic spherulitic carbon from poly-divinylbenzene by pressure hydrolysis. J. Mater. Sci. 1981, 16, 1989-1993.

(36) Guigo, N.; Mija, A.; Zavaglia, R.; Vincent, L.; Sbirrazzuoli, N. New insights on the thermal degradation pathways of neat poly(furfuryl alcohol) and poly(furfuryl alcohol)/ $\mathrm{SiO}_{2}$ hybrid materials. Polym. Degrad. Stab. 2009, 94, 908-913.

(37) Yao, J.; Wang, H.; Liu, J.; Chan, K.-Y.; Zhang, L.; Xu, N. Preparation of colloidal microporous carbon spheres from furfuryl alcohol. Carbon 2005, 43, 1709-1715.

(38) Men, X. H.; Zhang, Z. Z.; Song, H. J.; Wang, K.; Jiang, W. Functionalization of carbon nanotubes to improve the tribological properties of poly(furfuryl alcohol) composite coatings. Compos. Sci. Technol. 2008, 68, 1042-1049.

(39) Cheng, L. T.; Tseng, W. J. Effect of acid treatment on structure and morphology of carbons prepared from pyrolysis of polyfurfuryl alcohol. J. Polym. Res. 2010, 17, 391-399.

(40) Glover, T. G.; Dunne, K. I.; Davis, R. J.; LeVan, M. D. Carbon-silica composite adsorbent: Characterization and adsorption of light gases. Micropor. Mesopor. Mater. 2008, $111,1-11$.

(41) Príncipe, M.; Suárez, H.; Jimenez, G. H.; Martínez, R.; Spange, S. Composites prepared from silica gel and furfuryl alcohol with p-toluenesulphonic acid as the catalyst. Polym. Bull. 2007, 58, 619-626. 
(42) Shi, L.; Yao, J.; Jiang, J.; Zhang, L.; Xu, N. Preparation of mesopore-rich carbons using attapulgite as templates and furfuryl alcohol as carbon source through a vapor deposition polymerization method Micropor. Mesopor. Mater. 2009, 122, 294-300.

(43) Müller, H.; Rehak, P.; Jäger, Ch.; Hartmann, J.; Meyer, N.; Spange, S. A concept for the fabrication of penetrating carbon/silica hybrid materials. Adv. Mater. 2000, 12, 16711674.

(44) Nabais, J. M. V.; Carrott, P. J. M.; Ribeiro Carrott, M. M. L. From commercial textile fibres to activated carbon fibres: Chemical transformations. Mater. Chem. Phys. 2005, 93, 100-108.

(45) Rahaman, M. S. A.; Ismail, A. F.; Mustafa, A. A review of heat treatment on polyacrylonitrile fiber. Polym. Degrad. Stab. 2007, 92, 1421-1432.

(46) Jing, M.; Wang, C.; Wang, Q.; Bai, Y.; Zhu, B. Chemical structure evolution and mechanism during pre-carbonization of PAN-based stabilized fiber in the temperature range of 350-600². Polym. Degrad. Stab. 2007, 92, 1737-1742.

(47) Wangxi, Z.; Jie, L.; Gang, W. Evolution of structure and properties of PAN precursors during their conversion to carbon fibers. Carbon 2003, 41, 2805-2812.

(48) Vitkovskaya, R. F.; Rumynskaya, I. G.; Smirnov, A. Yu. Structural transformations in polyacrylonitrile during modification and fabrication of fibers and films with catalytic properties. Fibre Chem. 2008, 40, 202-205.

(49) Toda, M.; Takagaki, A.; Okamura, M.; Kondo, J. N.; Hajashi, S.; Domen, K.; Hara, M. Biodiesel made with sugar catalyst. Nature 2005, 438, 178. 
(50) Fang, L.; Zhang, K.; Li, X.; Wu, H.; Wu, P. Preparation of a carbon-silica mesoporous composite functionalized with sulfonic acid groups and its application to the production of biodiesel. Chinese J. Catal. 2012, 33, 114-122.

(51) Xing, R.; Liu, Y.; Wang, Y.; Chen, L.; Wu, H.; Jiang, Y.; He, M.; Wu, P. Active solid acid catalysts prepared by sulfonation of carbonization-controlled mesoporous carbon materials. Micropor. Mesopor. Mater. 2007, 105, 41-48.

(52) Nakajima, K.; Okamura, M.; Kondo, J. N.; Domen, K.; Tatsumi, T.; Hayashi, S.; Hara, M. Amorphous carbon bearing sulfonic acid groups in mesoporous silica as a selective catalyst. Chem. Mater. 2009, 21, 186-193.

(53) Liu, Y.; Chen, J.; Yao, J.; Lu, Y.; Zhang, L.; Liu, X. Preparation and properties of sulfonated carbon-silica composites from sucrose dispersed on MCM-48. Chem. Eng. J. 2009, 148, 201-206.

(54) Kruk, M.; Dufour, B.; Celer, E. B.; Kowalewski, T.; Jaroniec, M.; Matyjaszewski, K. Synthesis of mesoporous carbons using ordered and disordered mesoporous silica templates and polyacrylonitrile as carbon precursor. J. Phys. Chem. B 2005, 109, 9216-9225.

(55) Martín, A.; Morales, G.; van Grieken, R.; Cao, L.; Kruk, M. Acid hybrid catalysts from poly(styrenesulfonic acid) grafted onto ultra-large-pore SBA-15 silica using atom transfer radical polymerization. J. Mater. Chem. 2010, 20, 8026-8035.

(56) Li, C.; Yang, J.; Wang, P.; Liu, J.; Yang, Q. An efficient solid acid catalyst: Poly-pstyrenesulfonic acid supported on SBA-15 via surface-initiated ATRP. Micropor. Mesopor. Mater. 2009, 123, 228-233.

(57) Zhai, Y.; Tu, B.; Zhao, D. Organosilane-assisted synthesis of ordered mesoporous poly(furfuryl alcohol) composites. J. Mater. Chem. 2009, 19, 131-140. 
(58) Liu, Y.; Ju, M.; Wang, C.; Zhang, L.; Liu, X. Preparation of monodisperse mesoporous carbon microspheres from poly-(furfuryl alcohol)-silica composite microspheres produced in a microfluidic device. J. Mater. Chem. 2011, 21, 15049-15056.

(59) Gierszal, K. P.; Jaroniec, M. Large pore volume carbons with uniform mesopores and macropores: Synthesis, characterization, and relations between adsorption parameters of silica templates and their inverse carbon replicas. J. Phys. Chem. C 2007, 111, 9742-9748.

(60) Kruk, M.; Jaroniec, M.; Kim, T. W.; Ryoo, R. Synthesis and characterization of hexagonally ordered carbon nanopipes. Chem. Mater. 2003, 15, 2815-2823.

(61) Burket, C. L.; Rajagopalan, R.; Marencic, A. P.; Dronvajjala, K.; Foley, H. C. Genesis of porosity in polyfurfuryl alcohol derived nanoporous carbon. Carbon 2006, 44, 2957-2963.

(62) Chuang, I. S.; Maciel, G. E.; Myers, G. E. ${ }^{13}$ C NMR study in curing furfuryl alcohol resins. Macromolecules 1984, 17, 1087-1090.

(63) Smith, M. A.; Foley, H. C.; Lobo, R. F. A simple model describes the PDF of a non graphitizing carbon. Carbon 2004, 42, 2041-2048.

(64) Eckert, H.; Levendis, Y. A.; Flagant, R. C. Glassy carbons from poly(furfuryl alcohol) copolymers: structural studies by high-resolution solid state NMR techniques. J. Phys. Chem. 1988, 92, 5011-5019.

(65) Kumar, A.; Lobo, R. F.; Wagner, N. J. Porous amorphous carbon models from periodic Gaussian chains of amorphous polymers. Carbon 2005, 43, 3099-3111.

(66) Mariwala, R. K.; Foley, H. C. Evolution of ultramicroporous adsorptive structure in poly(furfuryl alcohol)-derived carbogenic molecular sieves. Ind. Eng. Chem. Res. 1994, 33, $607-615$. 
(67) Li, G.; Lu, Z.; Huang, B.; Wang, Z.; Huang, H.; Xue, R.; Chen, L. Raman scattering investigation of carbons obtained by heat treatment of a polyfurfuryl alcohol. Solid State Ionics 1996, 89, 327-331.

(68) Wang, Z.; Lu, Z.; Huang, Y.; Xue, R.; Huang, X.; Chen, L. Characterizations of crystalline structure and electrical properties of pyrolyzed polyfurfuryl alcohol. J. Appl. Phys. 1997, 82, 5705-5710.

(69) Kotdawala, R. R. Adsorption Studies of Hazardous Air Pollutants in Microporous Adsorbents using Statistical and Molecular Simulation Techniques. Ph.D. Dissertation, Worcester Polytechnic Institute, MA, 2007. 


\section{Figure captions}

Fig. 1. TG and DTG curves measured for the PFA/MCM-41 samples in the oxidizing atmosphere (A) and determined PFA loading in the studied composites (B)

Fig. 2. Adsorption-desorption isotherms (A) $\left(\mathrm{N}_{2}, 77 \mathrm{~K}\right)$ and NLDFT pore size distribution curves based on adsorption branch of isotherm (B) for the pristine support and the calcined PFA/MCM-41 composites (pristine MCM-41 (a) and PFA/MCM-41 samples thermally treated at $523 \mathrm{~K}$ with intended polymer $/ \mathrm{SiO}_{2}$ mass ratios of 0.1 (b), 0.4 (c), 1.0 (d) and $2.0(\mathrm{e}))$

Fig. 3. BET surface area and total pore volume of the parent support and thermally treated PFA/MCM-41 composites ( $\mathrm{V}_{\text {total }}$ determined by single point method at $p / p_{0}=0.98$; the intended PFA/MCM-41 mass ratios are declared above the bars)

Fig. 4. TEM images taken for pristine MCM-41 support (A) and PFA0.6/MCM-41 calcined at $523 \mathrm{~K}(\mathrm{~B})$

Fig. 5. MEK adsorption capacities of thermally treated bulky PFA and the PFA0.4/MCM-41 composite (A) and adsorption capacities of the composites partially carbonized at $523 \mathrm{~K}$ containing various amounts of polymeric species (B), measured by dynamic method (the intended PFA/MCM-41 mass ratios are declared above the bars)

Fig. 6. TG/DTG curves collected in inert atmosphere for bulky PFA and the PFA/MCM-41 composites containing various amounts of polymeric material (A) and FTIR maps of the gaseous products evolved during the PFA decomposition for neat PFA and the PFA0.4/MCM-41 and PFA1.0/MCM-41 samples in inert atmosphere (B)

Fig. 7. The proposed mechanism of thermal transformation of PFA deposited on MCM-41 

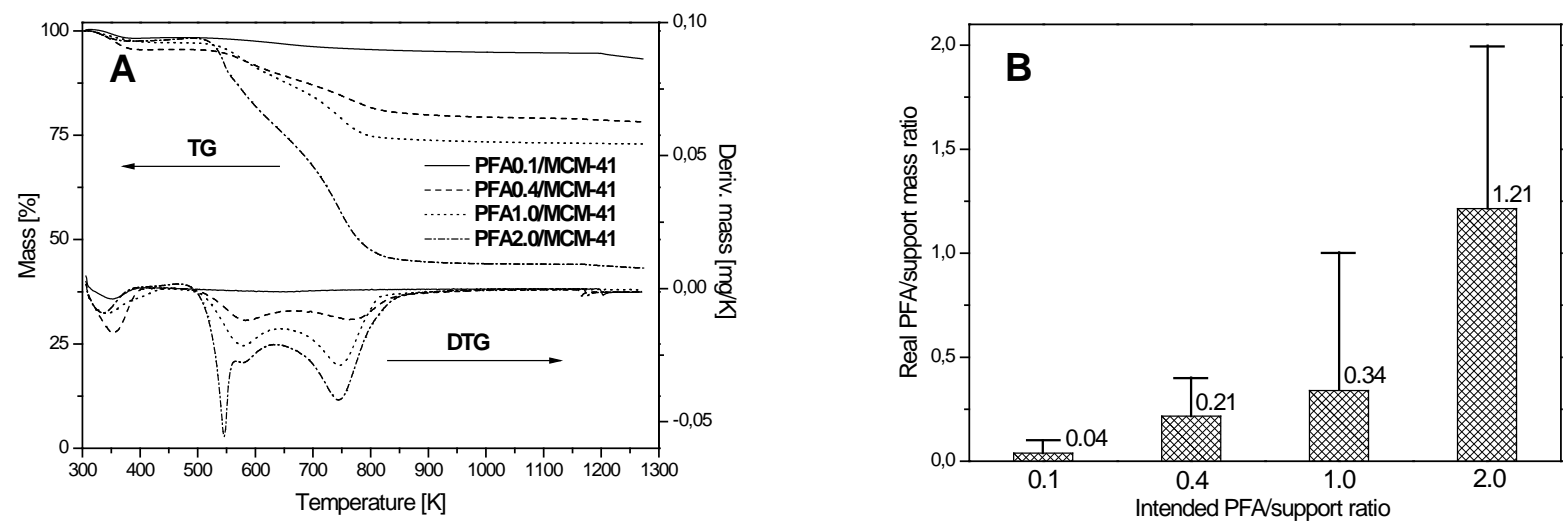

Fig. 1. Janus et al. 

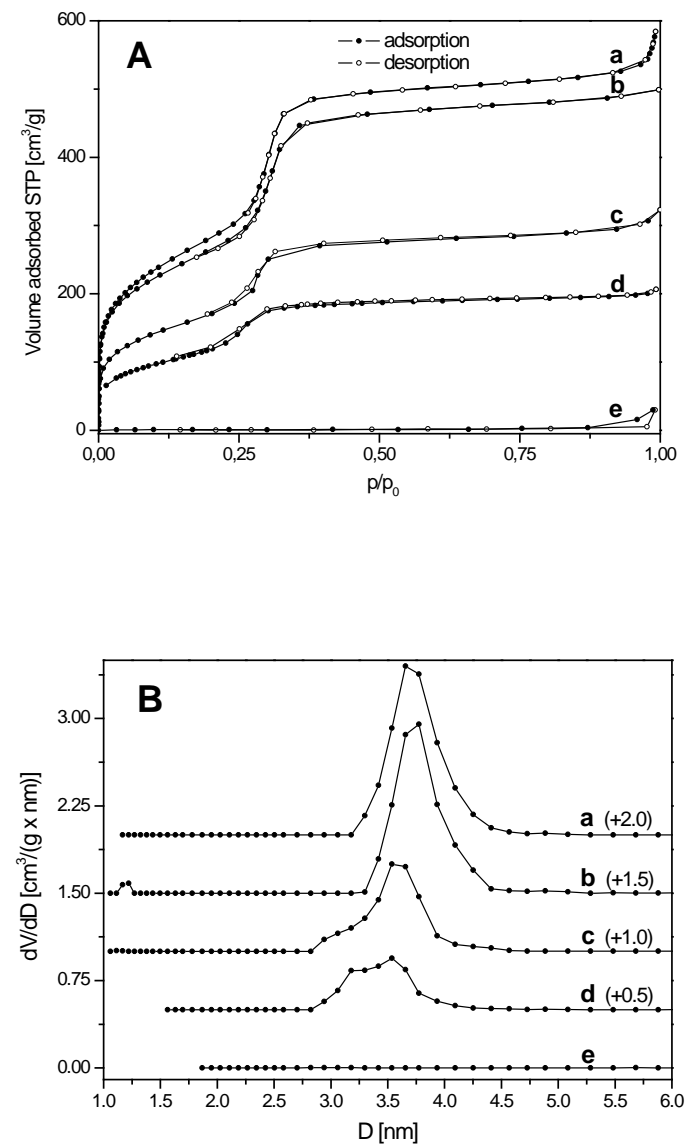

Fig. 2. Janus et al. 


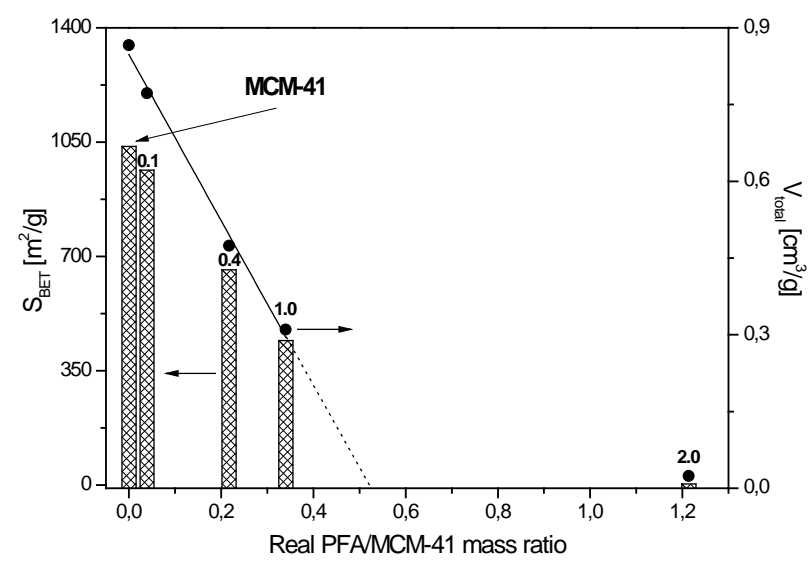

Fig. 3. Janus et al. 


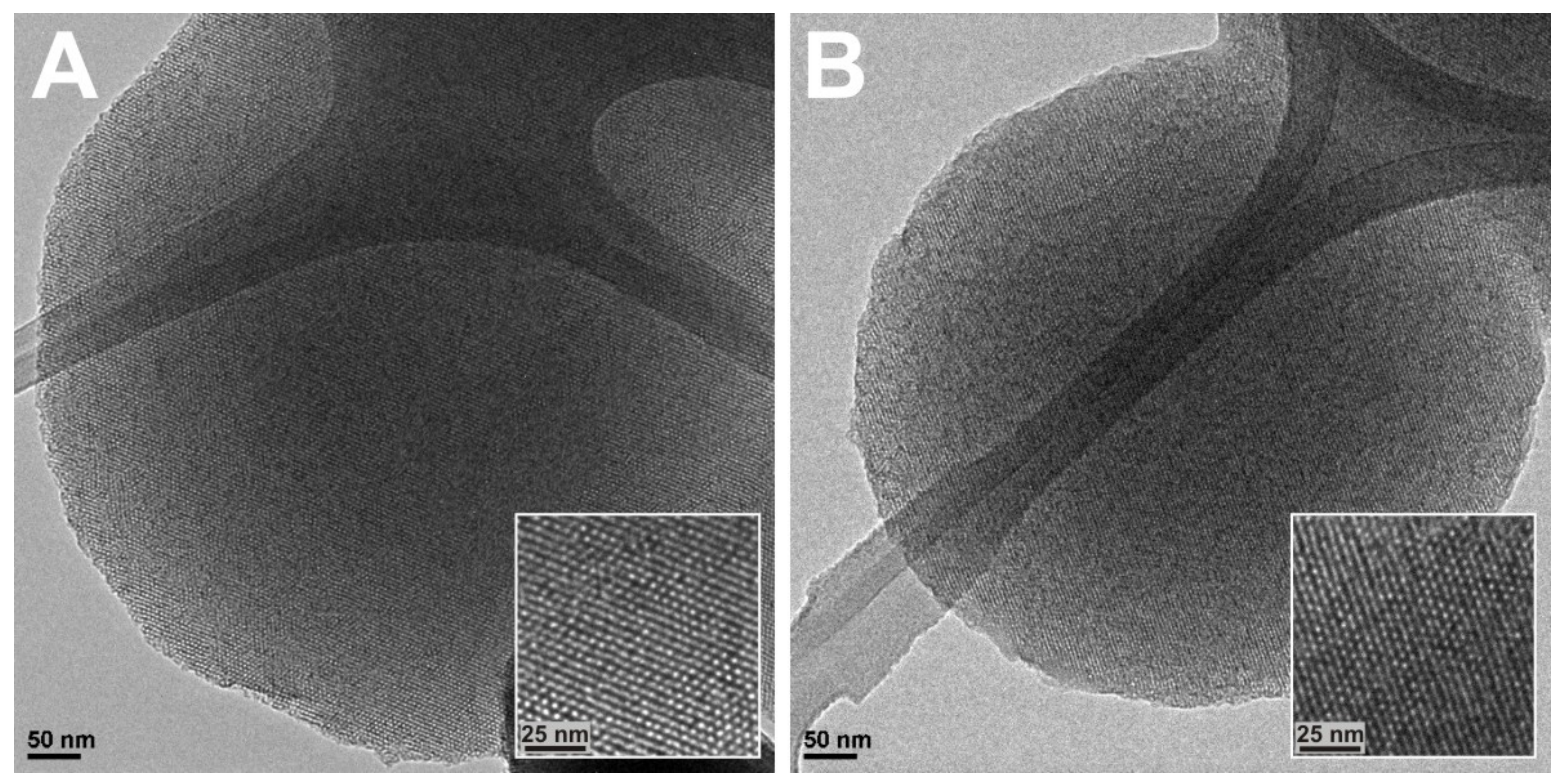

Fig. 4. Janus et al. 

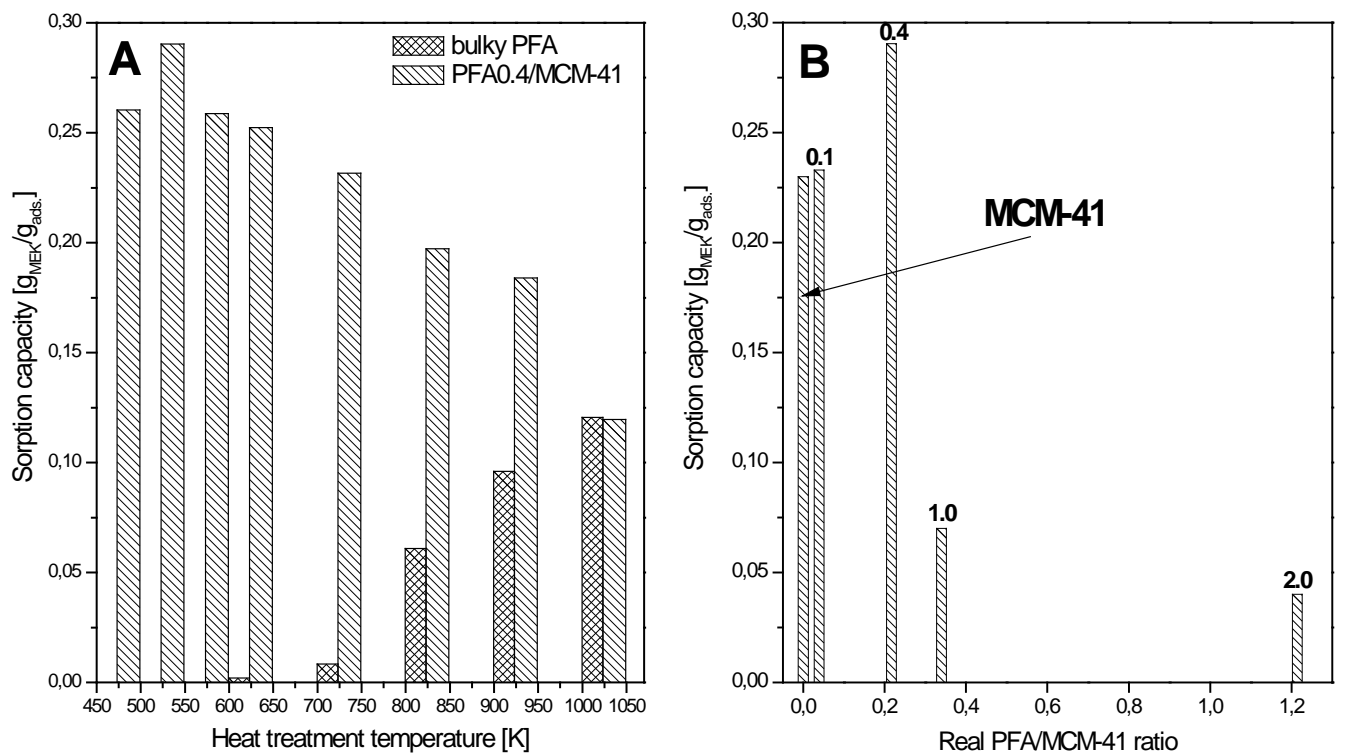

Fig. 5. Janus et al. 

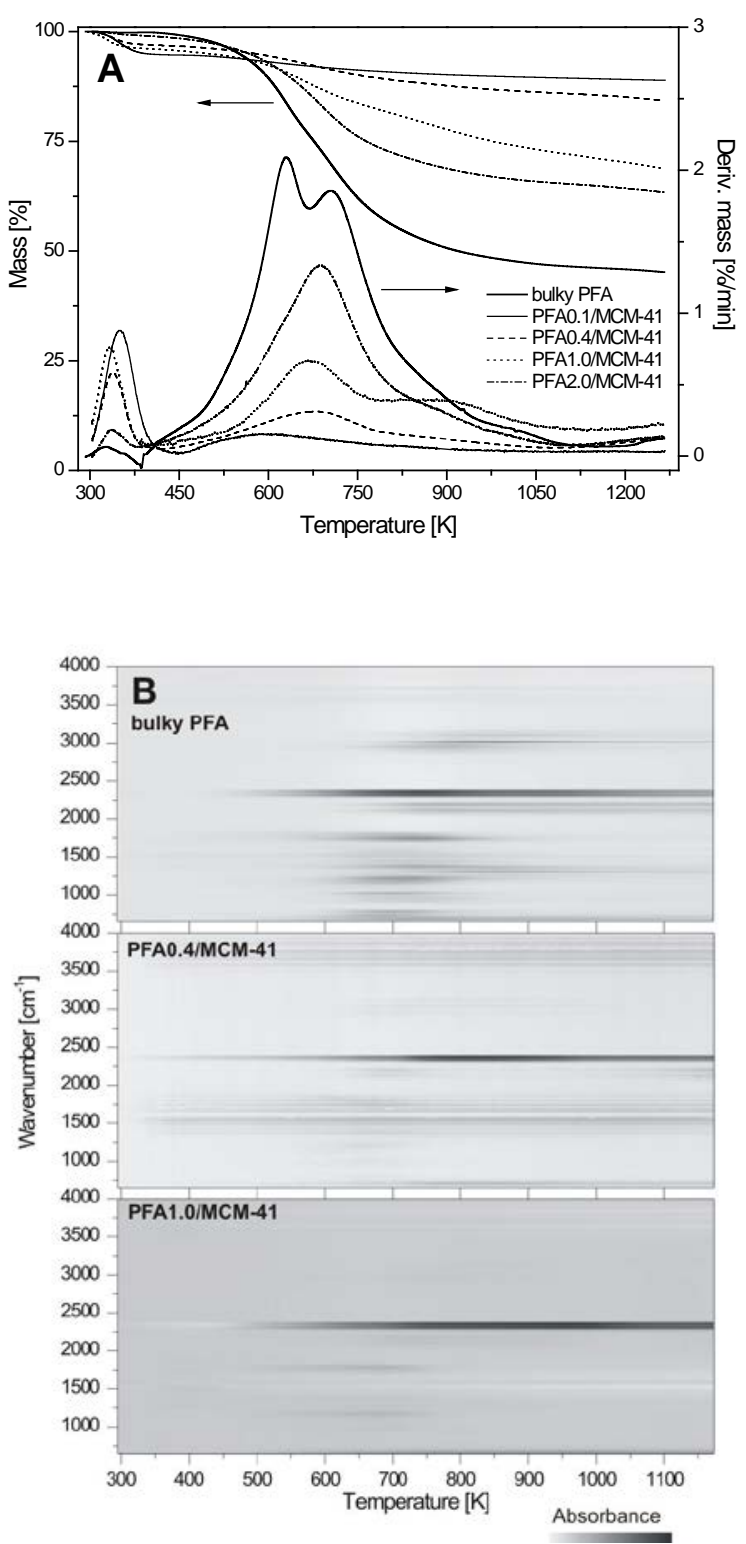

Fig. 6. Janus et al. 

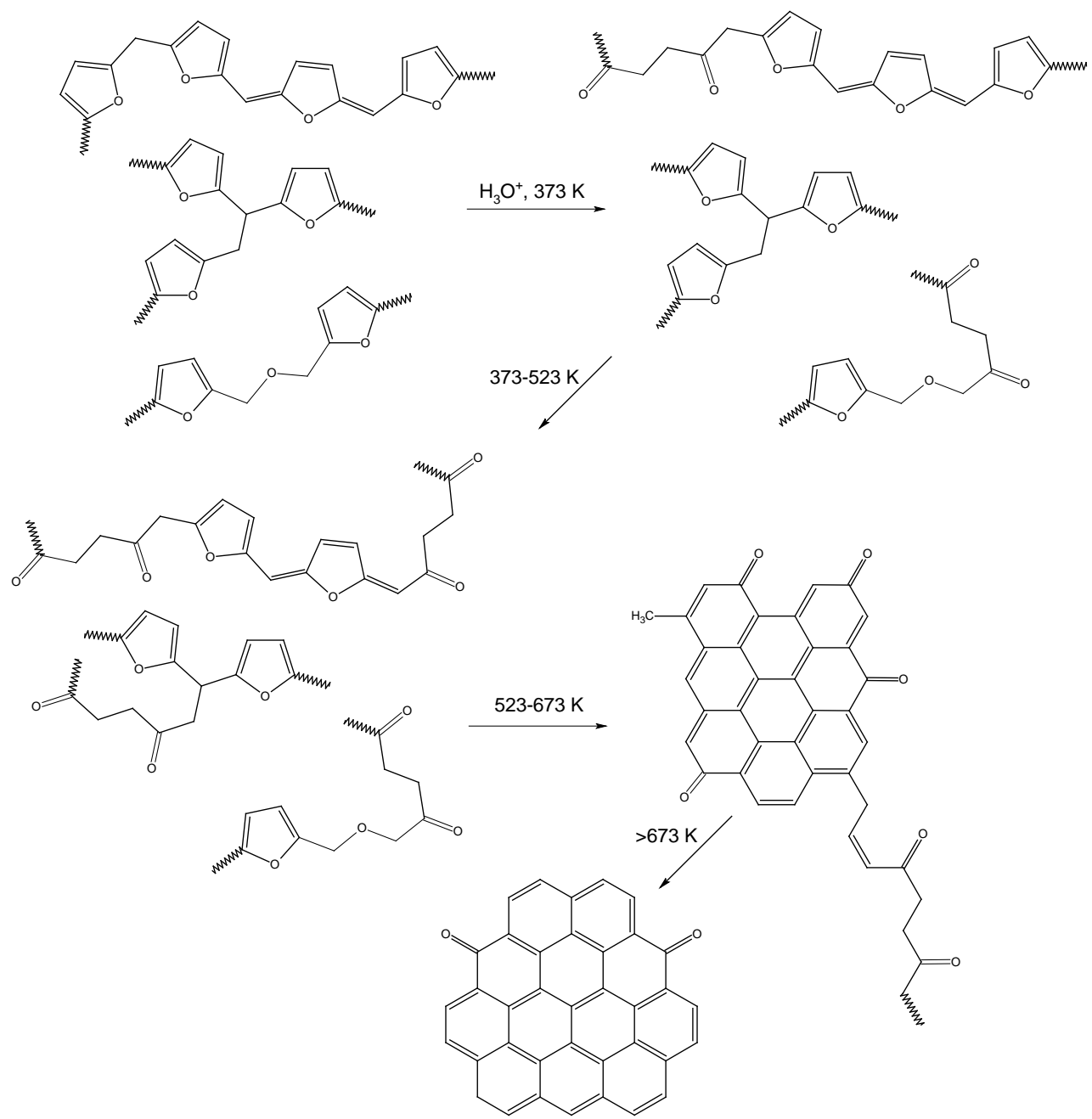

Fig. 7. Janus et al. 
Table 1. Atomic contributions of surface species detected in PFA0.4/MCM-41 (at. \%; binding energies given in brackets)

\begin{tabular}{|c|c|c|c|c|c|c|}
\hline \multirow[b]{2}{*}{ Sample } & \multirow{2}{*}{$\begin{array}{l}\text { Si } 2 p \\
\underline{\mathrm{SiO}}_{2} \\
(103.6 \mathrm{eV})\end{array}$} & \multicolumn{2}{|l|}{$\mathrm{O} 1 \mathrm{~s}$} & \multicolumn{3}{|l|}{$\mathrm{C} 1 \mathrm{~s}$} \\
\hline & & $\begin{array}{l}\mathrm{C}=\underline{\mathrm{O}} \\
(531.7 \pm 0.1 \mathrm{eV})\end{array}$ & $\begin{array}{l}-\underline{\mathrm{OH}}, \mathrm{Si}_{\underline{2}}, \mathrm{C}-\underline{\mathrm{O}}-\mathrm{C} \\
(532.9 \pm 0.2 \mathrm{eV})\end{array}$ & $\begin{array}{l}\underline{\mathrm{C}}=\mathrm{C}, \underline{\mathrm{C}}-\mathrm{C}, \underline{\mathrm{CH}}_{2} \\
(284.5 \pm 0.3 \mathrm{eV})\end{array}$ & $\begin{array}{l}\text { C-OH, } \underline{\mathrm{C}}-\mathrm{O} \\
(286.0 \mathrm{eV})\end{array}$ & $\begin{array}{l}\mathrm{C}=\mathrm{O} \\
(287.4 \pm 0.2 \mathrm{eV})\end{array}$ \\
\hline MCM-41 & 34.4 & - & 65.6 & - & - & - \\
\hline PFA0.4/MCM-41 & 25.0 & 1.3 & 52.7 & 15.9 & 3.7 & 1.3 \\
\hline PFA0.4/MCM-41 $473 \mathrm{~K}$ & 24.0 & 1.8 & 48.4 & 20.5 & 3.5 & 1.8 \\
\hline PFA0.4/MCM-41 523 K & 24.3 & 1.9 & 48.4 & 20.6 & 2.9 & 1.9 \\
\hline PFA0.4/MCM-41 $573 \mathrm{~K}$ & 25.1 & 1.4 & 48.8 & 20.7 & 2.6 & 1.4 \\
\hline PFA0.4/MCM-41 $673 \mathrm{~K}$ & 27.0 & 0.7 & 49.3 & 20.4 & 2.1 & 0.7 \\
\hline
\end{tabular}

